\title{
Effect of He-Injection on Irradiation Damage in Heat Affected Zone of Welded SUS304 Steel
}

\author{
Dong-Su Bae ${ }^{1}$, Hiroshi Kinoshita ${ }^{2}$, Tamaki Shibayama and Heishichiro Takahashi ${ }^{2}$ \\ ${ }^{1}$ Dept. of Materials and Metallurgical Engineering, Dong-Eui University, Busan 614-714, Korea \\ ${ }^{2}$ CARET, Hokkaido University, Sapporo 060-8628, Japan
}

\begin{abstract}
The heat affected zone (HAZ) of welded SUS304 steel has been irradiated using three irradiation modes of electron single irradiation, electron irradiation after He-injection and electron / He ion dual-beam irradiation by $1250 \mathrm{kV}$ high voltage electron microscope (HVEM) connected with an ion accelerator. Void size in the specimen with pre-injected helium increased considerably and the void size distribution was a bi-modal. Void swelling is the highest in the case of electron irradiation after He-injection. The segregation of solutes of Ni and Cr near grain boundary was suppressed in the cases of electron $/ \mathrm{He}^{+}$-ion dual-beam and electron irradiation after He-injection comparing with electron single irradiation.
\end{abstract}

(Received July 28, 2003; Accepted November 14, 2003)

Keywords: irradiation damage, electron $/ \mathrm{He}^{+}$-ion dual-beam irradiation, electron-beam irradiation after He-injection, 304 stainless steel, high voltage electron microscope, in-situ observation, heat affected zone, void swelling, segregation

\section{Introduction}

Nuclear power plant has operated at the last of 1970's in Korea, and development of the nuclear power plant has been required because of the increased demand for electrical power with industrial development. ${ }^{1)}$ However, it is difficult to newly construct a new nuclear power plant, because of a kind of 'not in my back yard' (NIMBY) phenomenon and environmental problem.

The important issue for nuclear reactor is how to extend safely the lifetime of the reactor. One of the better ways to accomplish these issues is to repair and /or to replace the degraded components of reactor materials. It has been considered welding is available technique for repairing reactors composed of steels such as SUS 304 and SUS304L stainless steels used as popular structural materials for nuclear reactor. ${ }^{2)}$ However, the characteristics such as mechanical and chemical properties of the heat affected zone (HAZ) are especially degraded due to irradiation hardening and accumulation of helium. Therefore it has been demanded to study the effects of irradiation on welded materials by considering helium atoms produced by a reaction of $(n, \alpha)$ in reactor. A lots of works have been performed regarding the effect of irradiation on microstructural evolution of austenitic stainless steels, ${ }^{3-14)}$ but the relation between helium introduced during irradiation and void nucleation etc. has not been clarified. The aim of present study is to make clear the helium effects on mainly void nucleation and growth for welded SUS304 stainless steel by means of simulation experiment using electron single, Electron $/ \mathrm{He}^{+}$-ion dual-beam and electron irradiation after He-injection.

\section{Experimental Procedure}

The chemical composition of SUS304 stainless steels used is shown in Table 1. The specimens were prepared from HAZ part of SUS304 stainless steels aged at $673 \mathrm{~K}$ for 1000 hours after tungsten inert gas (TIG) arc welding, and then disk foils
Table 1 Chemical composition of SUS304 steel(mass\%).

\begin{tabular}{ccccccc}
\hline & $\mathrm{C}$ & $\mathrm{P}$ & $\mathrm{S}$ & $\mathrm{Cr}$ & $\mathrm{Ni}$ & $\mathrm{Fe}$ \\
\hline SUS304 & 0.05 & 0.025 & 0.004 & 18.25 & 8.27 & bal. \\
\hline
\end{tabular}

with $3 \mathrm{~mm}$ diameter for TEM observation were punched out for TEM observation, and finally were electrochemically jet polished.

Electron single irradiation, electron $/ \mathrm{He}^{+}$-ion dual-beam and electron irradiation after He-injection were carried out at $673 \mathrm{~K}$ up to $5.4 \mathrm{dpa}$ for foils prepared from HAZ of welded SUS304 steel using 1,250 kV HVEM (JEM-ARM1300) connected with ion accelerator. The electron single irradiation was carried out with a damage rate of about $0.5 \times 10^{-3} \mathrm{dpa} / \mathrm{s}$ at $673 \mathrm{~K}$ up to doses of $5.4 \mathrm{dpa}$. Helium injection was done with $40 \mathrm{appm} / \mathrm{dpa}$ using ion accelerator with $250 \mathrm{keV}$ accelerating voltage. Dual-beam irradiations were performed by HVEM connected with a $250 \mathrm{keV}$ ion accelerator.

Irradiation-induced segregation for solutes was analyzed by an energy dispersive X-ray analyzer (EDX) equipped with a $200 \mathrm{kV}$ FE-TEM. The spot size used is $0.5 \mathrm{~nm}$. The void distribution and void swelling were measured by image analysis of the observed microstructures.

\section{Results and Discussion}

\subsection{Microstructure change and void size distribution}

Figure 1 shows the microstructural evolution of void formed during electron irradiation after He-injection at $673 \mathrm{~K}$. The voids in small size were formed from early stage of irradiation as shown in Fig. 1(a). These voids gradually grew without new void formation with increasing of irradiation dose as shown in Figs. 1(b), (c) and (d). The voids nucleated in the early irradiation stage become preferential sink sites for vacancies, thus most of these voids continue to grow with irradiation. 
(a)
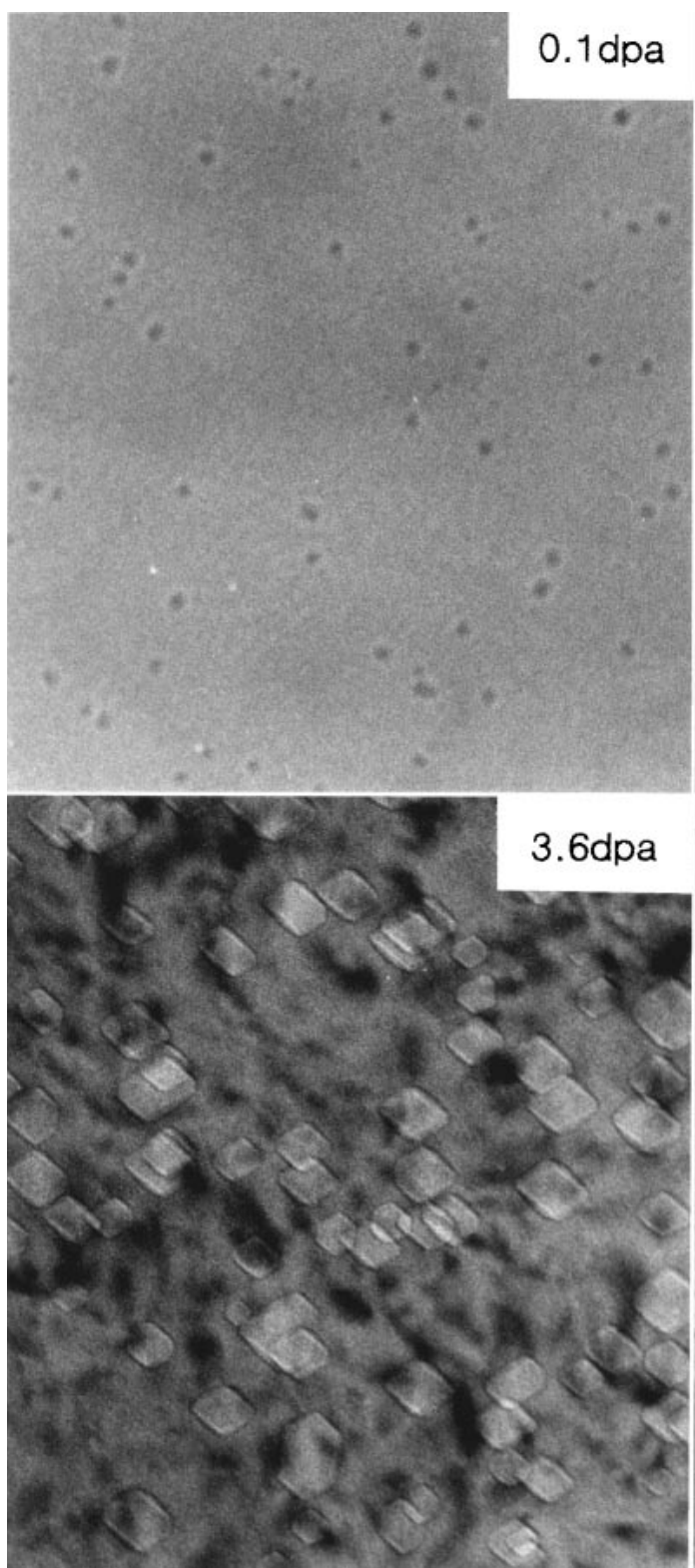

(c) (b)
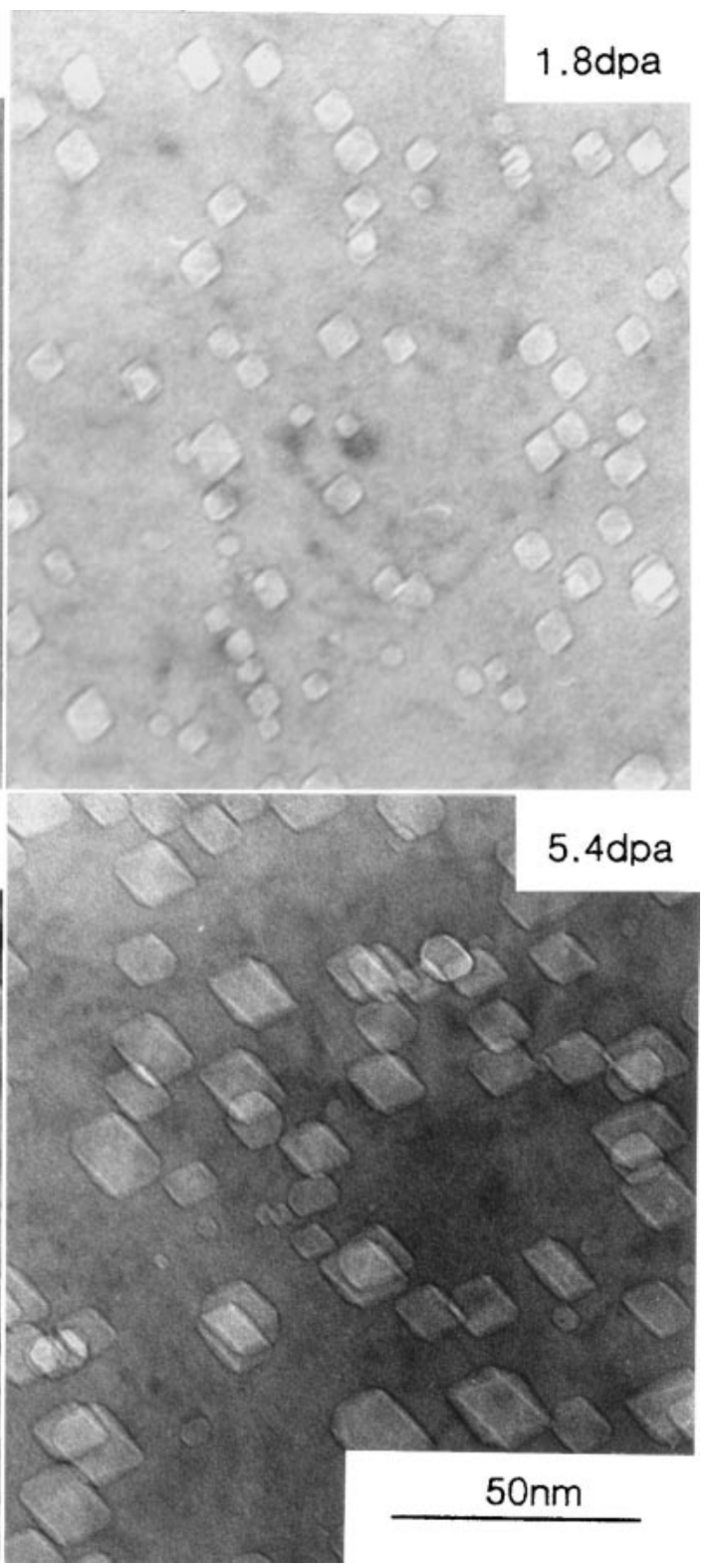

(d)

Fig. 1 Void formation changes under electron single irradiation up to (a) $0.1 \mathrm{dpa}$, (b) $1.8 \mathrm{dpa}$, (c) $3.6 \mathrm{dpa}$ and (d) $5.4 \mathrm{dpa}$ at $673 \mathrm{~K}$ after 216 appm He-injection in HAZ of SUS304 steel.

Figure 2 shows the size distribution of voids for each irradiation dose (dpa) in the specimen which was electronirradiated after He-injection at $673 \mathrm{~K}$. It is shown that a peak at $3.5 \mathrm{~nm}$ is observed at irradiation stage of $0.1 \mathrm{dpa}$ as shown in Fig. 2(a), and the void size distribution shows a monomodal one and the peak shifted to larger void size at $1.8 \mathrm{dpa}$ as shown in Fig. 2(b). The similar behavior has been reported for the electron single irradiation case. ${ }^{3)}$ This fact indicates that most of voids initially nucleated continue to grow monotonically with irradiation. However, with further irradiation to $3.6 \mathrm{dpa}$ the new peak appears at smaller void size, while the initially formed voids are growing, thus so-called bi-modal distribution with two peaks at different void size was observed (Fig. 2(c)). This bi-modal distribution is more clearly recognized at $5.4 \mathrm{dpa}$ as shown in Fig. 2(d). The bi- modal distribution is generally observed when helium gas is previously existed in the specimen, where the smaller size distribution may be attribute to the helium bubble with smaller size than that of critical diameter (corresponding to void size distribution of 'neck' part) to grow stable void. ${ }^{4)}$

The 'neck' part marked by arrow shifted to larger void size with irradiation dose although the critical void diameter is not usually changed with irradiation dose. These results indicate that at first voids are preferentially nucleated as an interaction of vacancies with injected helium, while interstitial dislocation loops are formed by agglomeration of interstitial atoms ${ }^{3)}$ in the early stage of irradiation. Therefore, the concentration of excess vacancies increases and smaller voids with helium grow by absorbing the excess vacancies introduced by electron irradiation. The voids in larger diameter than critical 


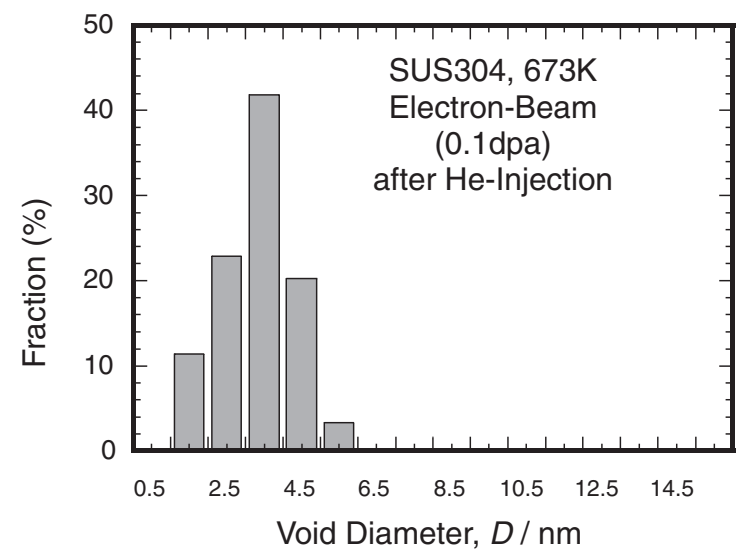

(a)

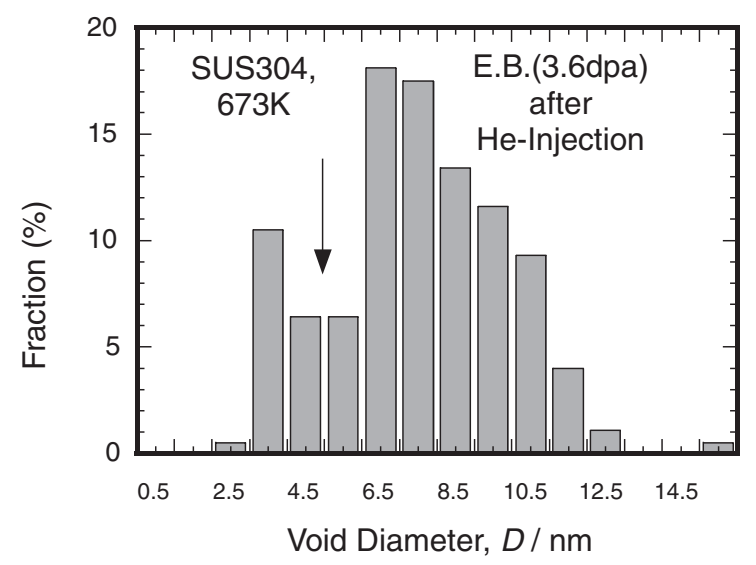

(c)

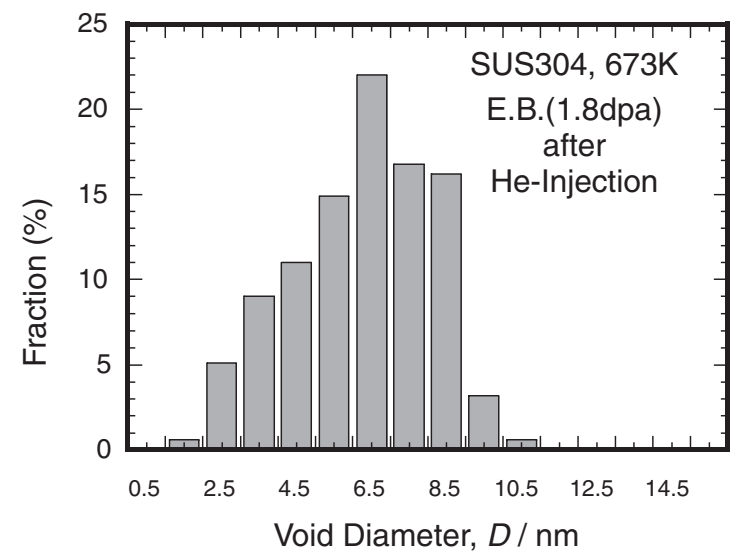

(b)

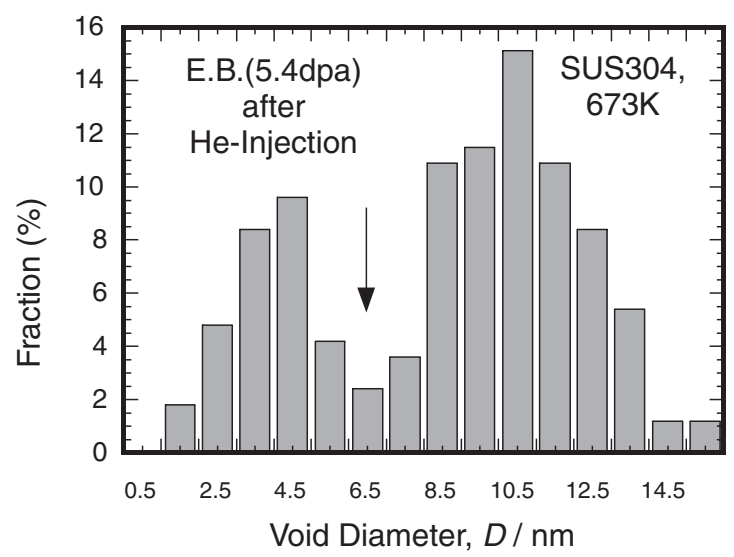

(d)

Fig. 2 Void size distributions of electron single irradiation up to (a) $0.1 \mathrm{dpa}$, (b) $1.8 \mathrm{dpa}$, (c) $3.6 \mathrm{dpa}$ and (d) $5.4 \mathrm{dpa}$ at $673 \mathrm{~K}$ after 216 appm He-injection in HAZ of SUS304 steel.

size continue to grow easily, while other smaller voids with helium than critical size remain separately, thus the bi-modal size distribution is observed in the growth process of initially nucleated voids.

Figure 3 shows the structures of void distribution after (a) electron single irradiation and (b) electrons $/ \mathrm{He}^{+}$-ions dualbeam irradiation up to $5.4 \mathrm{dpa}$ at $673 \mathrm{~K}$. The voids are formed in both dual-beam irradiation and electron single irradiation, and the grown larger voids and newly nucleated smaller voids are observed both for electron single irradiation as well as dual-beam irradiation conditions. On the other hand, the number density of voids is different depending on irradiation modes. When the specimen was irradiated by dual-beam with electrons/He ions, the void number density is higher than that of electron single irradiation. However, the bi-modal size distribution observed for electron irradiation after helium injection is not recognized and the distribution is monomodal being similar to the electron irradiation. ${ }^{3)}$ It is suggested from these results that void nucleation and its growth are continuously proceeded during irradiation by electron single irradiation and dual beam irradiation with electron and He ions. By comparison of two micro-structures in Fig. 3, it is observed that the largest voids formed by electron single irradiation are greater size than those for dual beam irradiation case. This size difference of larger voids might be attributed to helium effect injected, namely when helium atoms are simultaneously injected, the introduced vacancies interact with the helium simultaneously, then vacancy migration path becomes shorter, then vacancy diffusion is also retarded comparing with the case of electron single irradiation without helium.

\subsection{Void number density, void mean size and swelling}

Figures 4(a)-(c) show the void number density, void mean size and swelling for the HAZ of welded SUS304 steel for each irradiation mode as a function of irradiation dose. As being seen in Fig. 4(a) the void number density for electron irradiation after He-injection (Electron after He) is high from the initial irradiation stage and the number density is constant value of $10^{22} / \mathrm{m}^{3}$ even through irradiation dose increases. This could be explained by a model of vacancy trapping by the injected helium. Helium injected before electron irradiation could preferentially interact with vacancies introduced during electron irradiation so that helium acts as a nucleation site for void formation from the initial stage of irradiation and the nucleation of void is not newly caused by further irradiation, while more mobile interstitials agglomerate to form Frank type loops on the $\{111\}$ plane. ${ }^{10)}$ Thus, voids are nucleated with higher number density from lower irradiation dose ${ }^{11)}$ for the case of electron irradiation after He-injection.

On the other hand, the number density of voids is relatively lower at initial irradiation stage (about $10^{21} / \mathrm{m}^{3}$ ) and then 


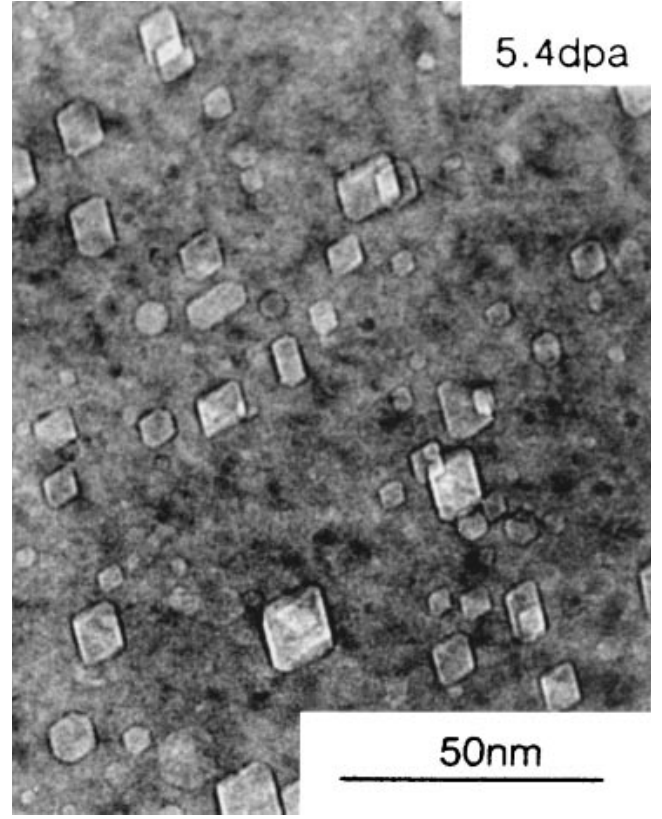

(a)

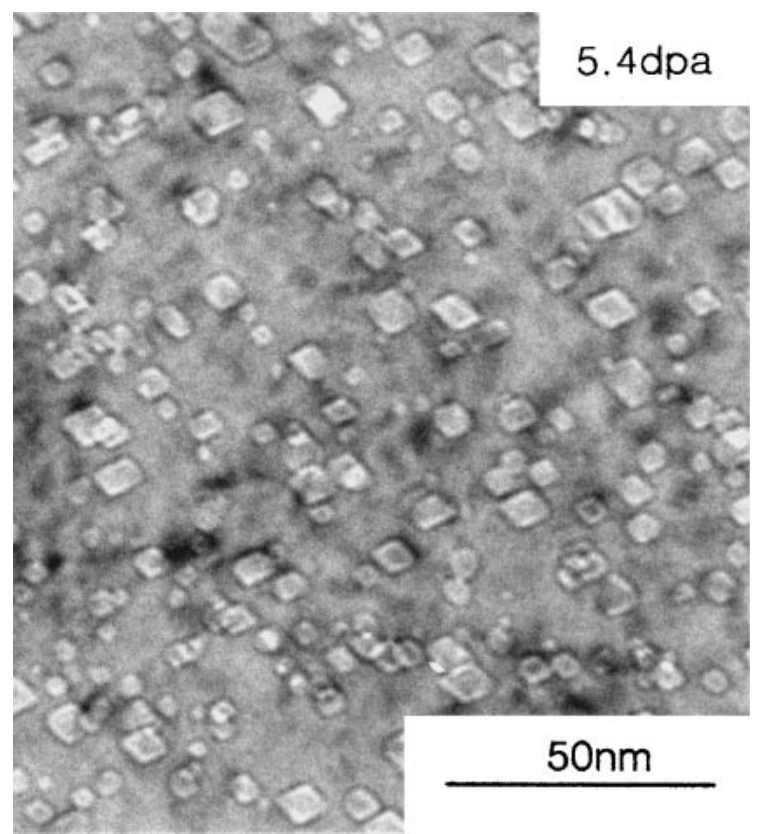

(b)

Fig. 3 Comparison of void formation for (a) electron single irradiation and (b) electrons $/ \mathrm{He}^{+}$-ion dual-beam irradiation to $5.4 \mathrm{dpa}$ at $673 \mathrm{~K}$ in $\mathrm{HAZ}$ of SUS304 steel.

gradually increase with increasing irradiation dose in the case of other two irradiation modes. The value for electron single irradiation (Electron) at higher dose tends to approach to the same value as the electron irradiation after He-injection, while in the case of dual-beam irradiation(Electron $+\mathrm{He}$ ), the number density becomes higher than that of electron irradiation over $2 \mathrm{dpa}$ and further increases with irradiation dose. These results indicate that the simultaneously injected helium atoms continuously act as nucleation site for void formation.

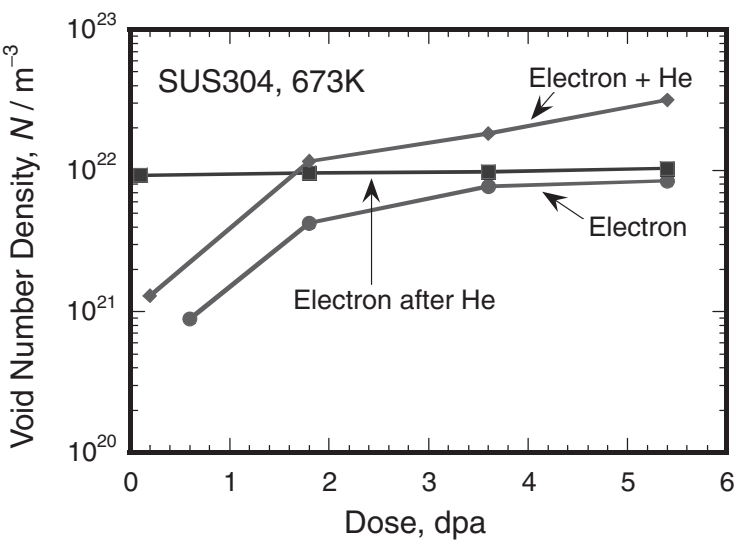

(a)

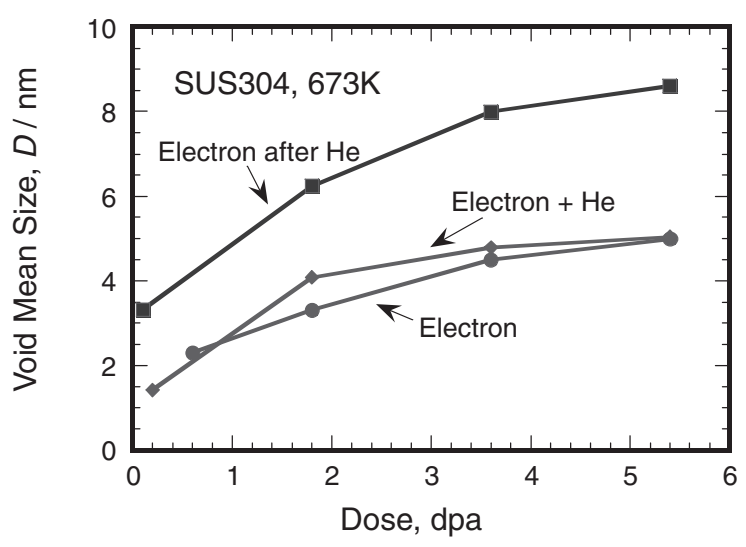

(b)

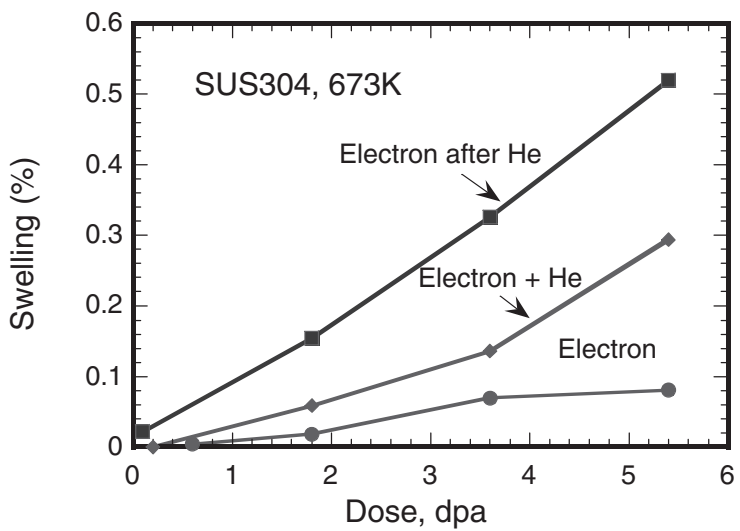

(c)

Fig. 4 (a) void number density, (b) void mean size and (c) swelling as a function of dpa for three types of irradiation mode, E.B.; electron single irradiation, He-E.B.; electron irradiation after He-injection, M.B.; electrons $/ \mathrm{He}^{+}$-ion dual-beam irradiation at $673 \mathrm{~K}$ in HAZ of SUS304 steel.

Figure 4(b) shows that the void mean size increases with increasing dose for electron irradiation after He-injection and dual-beam irradiation cases. The mean size does not changed largely for electron single irradiation. This trend for void growth is similar to that of dual-beam irradiation. The mean value of void size obtained from dual beam irradiation is much smaller than that of electron irradiation after Heinjection. It is also suggested from these results that helium injected previously has an important role for nucleation and growth of voids. Thus, behaviors of the point defects are 
obviously influenced by the existed helium atoms, which may exist in interstitial sites or substitutional lattice sites in alloys. When helium atoms occupy substitutional lattice sites and form $\mathrm{He}-\mathrm{V}$ pairs, these pairs act as a void nucleation site so that they promote void formation. ${ }^{11,15)}$

When comparing the swellings for each irradiation mode, the swellings at each dose are the highest for electron irradiation after He injection and then followed by dual beam irradiation and electron single irradiation. The swelling rate is also the highest for electron irradiation after He-injection as shown in Fig. 4(c), because of rapid growth of void by effective absorption of free vacancies introduced during electron irradiation.

\subsection{Irradiation-induced grain boundary segregation}

Figures 5(a)-(c) show the compositional concentration profile of $\mathrm{Cr}$ and Ni near a grain boundary after (a) electron single irradiation, (b) electron irradiation after He-injection and (c) dual-beam irradiation up to $5.4 \mathrm{dpa}$ at $673 \mathrm{~K}$ in HAZ of welded SUS304 steel. Equilibrium segregation occurs often during heat treatments of steels, but under irradiation condition non-equilibrium segregation is caused due to the interaction between solutes and point defects. The diffusion of solute atoms takes place by interaction of solutes with the fluxes of point defects to defect sinks such as grain boundary and void etc.

The phenomena of non-equilibrium segregation with enrichment of $\mathrm{Ni}$ and depletion of $\mathrm{Cr}$ at grain boundary has been reported for electron irradiation case for SUS316 steel ${ }^{12)}$ and SUS304 steel. ${ }^{3)}$ The atomic radius of $\mathrm{Cr}$ is larger than that of $\mathrm{Fe}$ based matrix in these stainless steels so that vacancies interact more effectively with $\mathrm{Cr}$ solute to relax the surrounding strain energy. In this case, $\mathrm{Cr}$ atoms preferentially diffuse by exchange mechanism with vacancy, thus the $\mathrm{Cr}$ atoms flow to opposite direction of vacancy flow. ${ }^{13)}$ That is, $\mathrm{Cr}$ concentration is depleted at grain boundary in the irradiated region, while $\mathrm{Cr}$ concentration increases in the area away from grain boundary.

On the other hand, $\mathrm{Ni}$ is undersized atom comparing to base matrix of the steels, therefore $\mathrm{Ni}$ atoms migrate to the same direction with interstitial point defect. Thus, the enrichment of $\mathrm{Ni}$ atoms occurs at grain boundary sink. Theses results obtained in present study coincide with previous results. ${ }^{12,14)}$

By Comparing with the electron irradiation after $\mathrm{He}$ injection and dual-beam irradiation, the void nucleation and swelling are lowest in the electron single irradiation. Therefore the void sink strength decreases in this irradiation condition. This means that point defects introduced during electron-irradiation can move longer mean free path without disappearing at internal sink sites such as voids, so the amount of defects migrating by interacting with solutes becomes to be great, namely the segregation of solute atoms is enhanced at the grain boundary.

On the other hand, when vacancies-He pairs become nucleation site for void formation as being observed for electron irradiation after $\mathrm{He}$-injection and dual beam irradiation cases, void sink strength increases so that the amount of point defects flow to grain boundary is decreased because of increment of disappearance of the defect at internal sinks.

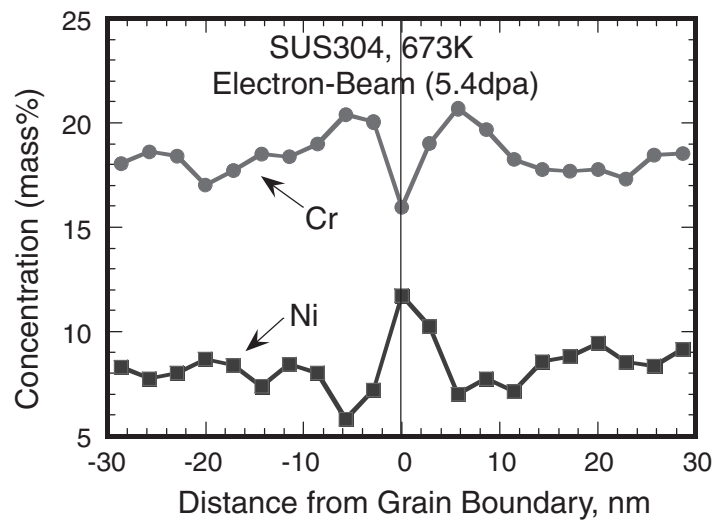

(a)

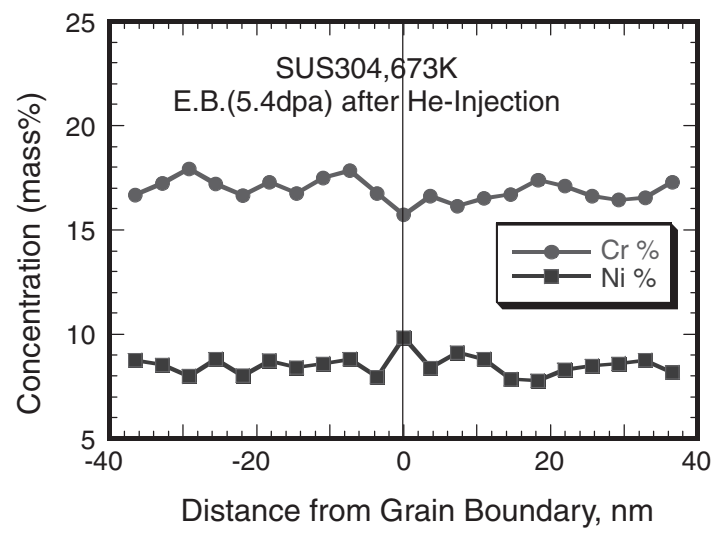

(b)

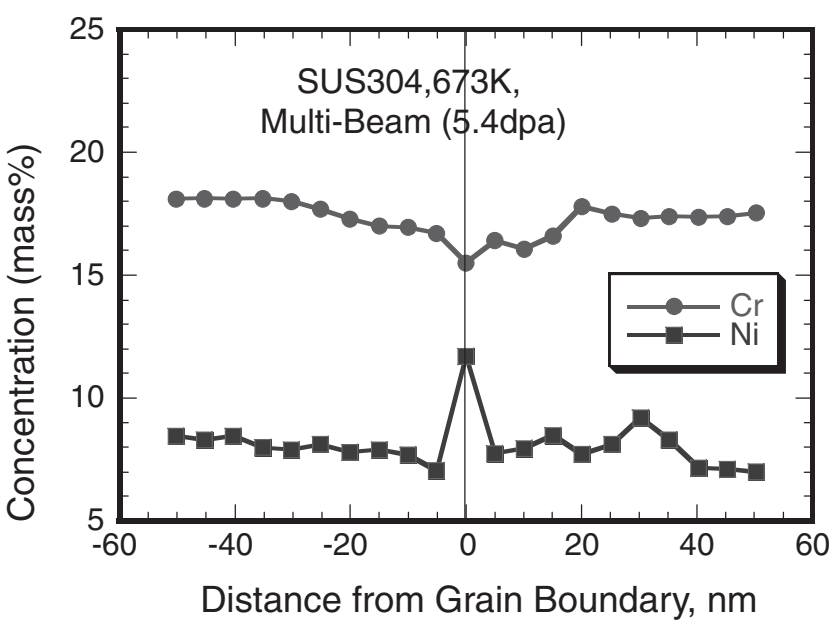

(c)

Fig. 5 Comparison of concentration profile near a grain boundary for (a) electron single irradiation, (b) electron irradiation after He-injection and (c) electrons $/ \mathrm{He}^{+}$-ion dual-beam irradiation up to $5.4 \mathrm{dpa}$ at $673 \mathrm{~K}$ in $\mathrm{HAZ}$ of SUS304 steel.

Consequently the amount of segregation at grain boundary is retarded.

\section{Conclusions}

Effect of He-injection on damaged microstructure of the heat affected zone in welded SUS304 steel was investigated by using the single electron-beam, electron-beam irradiation after He-injection and dual-beam irradiation. The results 
obtained are summarized as follows:

(1) Void size increases with irradiation dose in all of irradiation conditions. The void size at a given doses is larger in the case of electron irradiation after He-injection than other irradiation conditions because of enhanced void nucleation from the early irradiation stage.

(2) The void size distribution showed bi-modal one in the case of electron irradiation after He-injection at higher irradiation doses in the growth process of smaller voids nucleated as a result of interaction with helium from the early irradiation stage.

(3) Swelling rate of electron irradiation after He-injection is higher than that of other irradiation conditions, because of rapid growth of void by preferentially absorption of free vacancies introduced electron irradiation.

(4) Irradiation-induced segregations with the depletion of $\mathrm{Cr}$ and the enrichment of $\mathrm{Ni}$ were identified at grain boundaries. This segregation behavior was suppressed in the electron irradiation after He-injection and dual-beam irradiation compared to the case of electron single irradiation in the HAZ of welded SUS304 steel as a result of decrement of effective point defects concentration associated with solutes, because of increasing internal sink strength.

\section{Acknowledgements}

This work was supported by the Core University Program between Korea and Japan through Korea Science \& Engineering Foundation (KOSEF) and Japan Society for the Promotion of Science (JSPS). And also supported by
Research Center for Electronic Ceramics (RCEC) of DongEui University, which is supported by Busan Metropolitan City and KOSEF under Korea Ministry of Science and Technology (MOST).

\section{REFERENCES}

1) NEUTOPIA-News of Korean Nuclear Society 33 (2001) 5.

2) Department of Large Tokamaku Research: JAERI, JAERI-M-85-177.

3) D. S. Bae, H. Kinoshita and H. Takahashi: J. Nucl. Mater. 302 (2002) 60-65.

4) S. Ohnuki, H. Takahashi, Y. Hidaka and K. Katayama: Microsc. Microanal. Microstruct. 4 (1993) 313-322.

5) C. H. Woo, B. N. Singh and A. A. Semenov: J. Nucl. Mater. 239 (1996) $7-23$.

6) N. Akasaka, I. Yamagata and S. Ukai: J. Nucl. Mater. 283-287 (2000) 169-173.

7) R. Schublin and M. Victoria: J. Nucl. Mater. 283-287 (2000) 339-343.

8) I. J. O'Donnell, H. Huthmann and A. A. Tavassoli: Int. J. Pres. Ves. \& Piping 65 (1996) 209-220.

9) H. Takahashi and N. Hashimoto: Mater. Trans., JIM 34 (1993) 10271030.

10) K. Urban and M. Wilkens: Phys. Status Solid(a) 6 (1971) 173-185.

11) B. Hu, H. Kinoshita, T. Shibayama and H. Takahashi: Mater. Trans. 43 (2002) 622-626.

12) S. Ohnuki, H. Takahashi and T. Takeyama: Proc. 4th JIM Int. Symp. on Grain Boundary Structure and Related Phenomena, Supplement to Trans. JIM, Vol. 27 (1986) 625-632.

13) T. R. Anthony: In Radiation-Induced Void in Metals, Corbett. J. W. and Ianniello. L. C. (eds.) (1972) pp. 630-646.

14) P. R. Okamoto and L. E. Rehn: J. Nucl. Mater. 83 (1979) 2-7.

15) M. Kiritani, N. Yoshida and H. Tanaka: J. Phys. Soc. Jpn. 38 (1975) 1677-1683. 